\title{
Low-frequency variability of a two-layer ocean forced by periodic winds
}

\author{
Toshihiro Sakamoto \\ Department of Earth and Planetary Science, Graduate School of Science, The University of Tokyo, Tokyo 113-0033, Japan
}

(Received September 29, 2005; Revised December 20, 2005; Accepted April 17, 2006; Online published September 29, 2006)

\begin{abstract}
To seek the variability of the oceanic subtropical gyre on interannual and longer time scales we have conducted numerical experiments with a two-layer quasigeostrophic model in a square basin bounded by no-slip walls. We find that when the amplitude of annually periodic wind forcing is increased, the time series of the total energy exhibit a transition to chaos in such a manner that the response frequency constitutes a quasi-devil's staircase against the forcing amplitude; in particular, the $n$-cycles appear in descending order of $n$. The low-frequency modes may thus be produced by seasonal winds. Since, however, the power of the subharmonics is much weaker than that with the forcing frequency, their energy would be concealed by noise in the presence of stochastic wind forcing. The present result is in contrast with the case of the time-independent forcing in which we observe the intrinsic frequencies probably associated with the wave propagation, frequency locking and a cascade of period-doubling bifurcations.
\end{abstract}

Key words: Ocean circulation, dynamics, variability, subharmonics, devil's staircase, route to chaos.

\section{Introduction}

This article is intended as a contribution on nonlinear phenomena of the large-scale oceanic flows. However, since the regular readers of EPS might be relatively less interested in ocean dynamics, we review first some selected aspects of nonlinearity pertinent to this subject matter and then present the motivation of the present study.

\subsection{Nonlinear processes in large-scale ocean circula- tion}

A steady state of the subtropical gyre may be described in the simplest form by the linear theory that consists of a southward flow in a broad region called the Sverdrup interior and a frictional western boundary current (WBC) corresponding to the Kuroshio and the Gulf Stream; see Hendershott (1987) for a review. The nonlinear effects first considered in this context are associated with the advection in the WBC. In the 1960s perturbation analyses with the aid of numerical solutions confirmed an inertial recirculation and standing long planetary waves that are attached to the WBC (cf. Fig. 1); these nonlinear ingredients serve as an additional dissipation mechanism in the basin-wide balance of potential vorticity. An attempt was also made to address the same problem in the opposite direction, by starting with a purely inertial gyre and then treating friction as a perturbation (Hendershott, 1987). At any rate, the question is still open as to how such highly inertial boundary layers are matched with the Sverdrup interior. The nonlinearity in the WBC has also stimulated the instability theories (e.g. Orlanski, 1969) and the separation problems (e.g. Sakamoto (2002) for a review).

Vortex stretching may be responsible for another type

Copyright (c) The Society of Geomagnetism and Earth, Planetary and Space Sciences (SGEPSS); The Seismological Society of Japan; The Volcanological Society of Japan; The Geodetic Society of Japan; The Japanese Society for Planetary Sciences; TERRAPUB. of nonlinearity which led to the innovation of the classical general ocean circulation theory in the 1980s (Pedlosky, 1990). Strong stretching in the real ocean is inferred by the large displacements of the isopycnal surfaces above the main thermocline. Such features may be associated with finite-amplitude planetary waves (Anderson and Killworth, 1979; Willmott, 1985) that may evolve into a planetary shock front (Dewar, 1991; Sakamoto, 1999). In extreme cases some isopycnals are exposed to the atmosphere, so that motions can directly be driven on those isopycnal surfaces (Luyten et al., 1983). Even moderate stretching may create in the Sverdrup interior a region bounded by closed characteristics in which subsurface motions are induced by only minute vertical transfers of eddy momentum (Rhines and Young, 1982). Again, however, we have not completely settled the question as to whether such subsurface motions remain uncontaminated by the WBC (Sakamoto, 2001).

Numerical studies of unsteady problems have greatly widened our perspectives of the oceanic nonlinear processes. Since the 1970 s, the eddy-resolving calculations with simple layered ocean models, together with the deliberately designed field measurements, have unveiled the ubiquity of mesoscale eddies in the open oceans and their roles in controlling the large-scale, long-term mean motions (Holland and Lin 1975; Holland et al., 1983). In particular, energy budget analysis, originally developed in dynamic meteorology (Lorenz, 1955), has been employed systematically to investigate the degree of barotropic and/or baroclinic instabilities in various ocean circulation models. Verifying and seeking better parameterizations of eddy processes, e.g. the down-gradient diffusion of potential vorticity, has been one of the important subjects for improving coarse-grid models (Young, 1987; Gent and McWilliams, 1990). 
Table 1. Some numerical studies of the low-frequency variability of the wind-driven ocean circulation in relation to successive bifurcations.

\begin{tabular}{|c|c|c|c|c|c|c|}
\hline Study & Model & Gyre & $\begin{array}{c}\text { Basin size } \\
\text { EW }(\mathrm{km}) \times \mathrm{NS}(\mathrm{km})\end{array}$ & $\begin{array}{l}\text { Boundary } \\
\text { condition }\end{array}$ & Forcing & Control parameter \\
\hline CGIL01 & barotropic & double & $2560 \times 2560$ & free-slip & steady & forcing magnitude \\
\hline $\begin{array}{l}\text { VSCD02 } \\
\text { SD02 }\end{array}$ & barotropic QG & double & $1000 \times 1000$ & $\begin{array}{l}\text { no-slip }+ \\
\text { free-slip }{ }^{\dagger}\end{array}$ & steady & eddy viscosity \\
\hline JJG95 & 1.5-layer SW & double & $1000 \times 2000$ & no-slip & steady & forcing magnitude \\
\hline MH96 & 1.5-layer QG & double & $3600 \times 2800$ & free-slip & steady & forcing magnitude* \\
\hline $\mathrm{P} 02$ & 1.5-layer QG & double & $3600 \times 2800$ & free-slip & steady & biharmonic viscosity \\
\hline ND01 & 2-layer SW & double & $1000 \times 2000$ & no-slip & steady & forcing magnitude \\
\hline DK97 & $\left\{\begin{array}{l}\text { 1.5-layer QG } \\
\text { 2-layer QG }\end{array}\right.$ & double & $1000 \times 1000$ & $\begin{array}{l}\text { no-slip }+ \\
\text { free-slip }{ }^{\dagger}\end{array}$ & steady & eddy viscosity \\
\hline BM99 & $\left\{\begin{array}{l}\text { 1.5-layer QG } \\
\text { 2-layer QG }\end{array}\right.$ & double & $3840 \times 3840$ & no-slip & steady & eddy viscosity \\
\hline M00 & $\left\{\begin{array}{l}\text { barotropic } \\
\text { 2-layer QG }\end{array}\right.$ & double & $1024 \times 2048$ & no-slip & steady & eddy viscosity \\
\hline GFS02 & 2-mode QG & double & $1280 \times 2560$ & partial-slip & steady & $\begin{array}{l}\text { forcing magnitude } \\
\text { deformation radius }\end{array}$ \\
\hline KSPB95 & barotropic & single & $2000 \times 2000$ & $\begin{array}{l}\text { no-slip }+ \\
\text { free-slip }{ }^{\ddagger}\end{array}$ & steady & eddy viscosity \\
\hline MB97 & barotropic & single & $1024 \times 512$ & no-slip & steady & eddy viscosity \\
\hline BM97 & 1.5-layer QG & single & $800 \times 400$ & no-slip & steady & $\left\{\begin{array}{l}\text { eddy viscosity } \\
\text { deformation radius }\end{array}\right.$ \\
\hline BM98 & 2-layer QG & single & $800 \times 400$ & no-slip & steady & eddy viscosity \\
\hline S04 & 2-layer QG & single & $1000 \times 1000$ & no-slip & steady & forcing magnitude \\
\hline This study & 2-layer QG & single & $1000 \times 1000$ & no-slip & periodic & forcing magnitude \\
\hline
\end{tabular}

CGIL01: Chang et al. (2001), VSCD02: van der Vaart et al. (2002), SD02: Simonnet and Dijkstra (2002), JJG95: Jiang et al. (1995), MH96: McCalpin and Haidvogel (1996), P02: Primeau (2002), ND01: Nauw and Dijkstra (2001), DK97: Dijkstra and Katsman (1997), BM99: Berloff amd McWilliams (1999), M00: Meacham (2000), GFS02: Ghil et al. (2002), KSPB95: Kamenkovich et al. (1995), MB97: Meacham and Berloff (1997), BM97: Berloff and Meacham (1997), BM98: Berloff and Meacham (1998), S04: Sakamoto (2004). † Free-slip at the eastern and western boundaries and no-slip at the northern and southern boundaries. $\ddagger$ No-slip at the eastern and western boundaries and free-slip at the northern and southern boundaries. $*$ Together with the degree of asymmetry in the horizonal distribution of wind forcing.

\subsection{Toward an understanding of the ocean climate}

Although observational evidence is far from convincing, there has been a growing recognition that the temperate to mid-latitude North Pacific Ocean reveals the low-frequency variability in the sea-surface temperatures, large-scale flow patterns, and so forth, over a wide range of time scales (Miller et al., 1994; Trenberth and Hurrell, 1994). It is also known that the Kuroshio off Japan and its extension show interannual and decadal variations in its path, velocity and volume transport (Qiu and Joyce, 1992; Deser, 1999; Qiu, 2003). Since the oceanic motion above the main thermocline is mainly wind-driven, it is natural to raise the question as to how such low-frequency signals are produced in those regions where seasonal winds are predominant. To address such problems, extensive modeling efforts have been made from different points of view (Sura $e t$ al., 2000). Among the models proposed as a potential mechanism of the low-frequency variability are thermal forcing (Auad, 2003; Thompson and Ladd, 2004), stochastic wind forcing (Qiu, 2003; Hasselmann, 1976; Frankignoul et al., 1997; Cessi and Louazel, 2001) and the feedback to the atmosphere and vice versa (Pierce et al., 2001; Schneider et al., 2002).

Since the 1990s, a modern dynamical systems approach has been applied to the multiple equilibria and variability
Table 2. Model parameters.

\begin{tabular}{cl}
\hline Parameter & \multicolumn{1}{c}{ Value } \\
\hline$L$ & $1000 \mathrm{~km}$ \\
$H_{1}$ & $1000 \mathrm{~m}$ \\
$H_{2}$ & $3000 \mathrm{~m}$ \\
$f_{0}$ & $7.3 \times 10^{-5} \mathrm{~s}^{-1}$ \\
$\beta$ & $2 \times 10^{-11} \mathrm{~m}^{-1} \mathrm{~s}^{-1}$ \\
$\rho_{0}$ & $1000 \mathrm{~kg} \mathrm{~m}^{-3}$ \\
$g^{\prime}$ & $0.02 \mathrm{~m} \mathrm{~s}^{-2}$ \\
$A_{\mathrm{H}}$ & $150 \mathrm{~m}^{2} \mathrm{~s}^{-1}$ \\
\hline
\end{tabular}

of the large-scale wind-driven ocean circulation to explore the possibilities of describing the natural ocean climate by accessible models (Dijkstra, 2000). Along the same lines of research, we have shed light on transitions between different dynamic states of an oceanic gyre in order to estimate the extent to which those long-term variations are attributable to the internal nonlinear processes. Some previous studies are summarized in Table 1. Here, attention is focused on those studies using either the shallow-water (SW) or the quasigeostrophic (QG) layered model within a rectangular basin, rather than more elaborate models (e.g. Dijkstra 
and Weijer, 2005). These layered models are much simpler than the existing influential ocean climate models but may nevertheless be so complicated that the behavior of the output signals would be very different from that of some wellknown low-dimensional dynamical systems. The doublegyre experiments in the table include the counter-rotating subtropical and subpolar gyres separated by a free eastward jet which is absent from the single-gyre experiments. We note that in most cases the basin is much smaller than the world oceans, primarily because of the limited computational resources. In many of the previous studies the boundary condition is either free-slip or no-slip; the difference might be crucial in determining the behavior of the model ocean. The present study is a direct continuation of Sakamoto's (2004) single-gyre experiment by replacing the steady forcing with periodically changing forcing.

\section{Model}

In this study the stratified ocean is represented by a twolayer incompressible fluid with mean thickness $H_{1}$ and $H_{2}$ and constant density $\rho_{1}$ and $\rho_{2}$, where the subscript 1 and 2
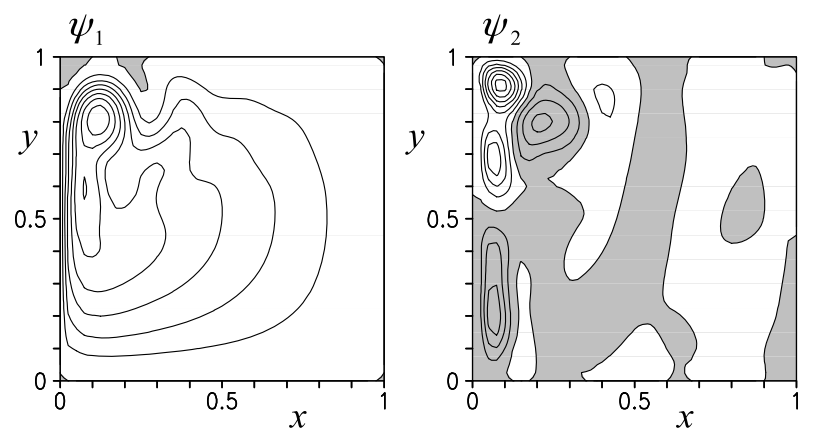

Fig. 1. Annual-mean streamfunctions $\psi_{1}$ (top) and $\psi_{2}$ (bottom) at year 801 for $\tau_{0}=0.079 \mathrm{~N} \mathrm{~m}^{-2}$. Contour intervals are $2000 \mathrm{~m}^{2} \mathrm{~s}^{-1}$ for $\psi_{1}$ and $100 \mathrm{~m}^{2} \mathrm{~s}^{-1}$ for $\psi_{2}$. Both the abscissa and the ordinate are scaled by $1000 \mathrm{~km}$. Regions where the streamfunction takes on negative values are shaded. denote the upper and lower layers, respectively. The ocean is located on a $\beta$-plane $0 \leq x \leq L, 0 \leq y \leq L$, where $x$ and $y$ are the eastward and northward coordinates, respectively. The surface and the bottom of the ocean are assumed to be flat. To further simplify the mathematical model, we assume the QG approximation (e.g. Pedlosky, 1987) in which 2-D flow in each layer is nondivergent to leading order, so that we can define the velocity streamfunction for each layer, $\psi_{1}$ and $\psi_{2}$, at all times. We thus consider a set of potential vorticity equations of the form

$$
\begin{aligned}
\frac{d q_{1}}{d t} & =A_{\mathrm{H}} \nabla^{4} \psi_{1}+\frac{1}{\rho_{0} H_{1}} W, \\
\frac{d q_{2}}{d t} & =A_{\mathrm{H}} \nabla^{4} \psi_{2}, \\
q_{1} & =\nabla^{2} \psi_{1}+\frac{f_{0}^{2}}{g^{\prime} H_{1}}\left(\psi_{2}-\psi_{1}\right)+\beta y, \\
q_{2} & =\nabla^{2} \psi_{2}-\frac{f_{0}^{2}}{g^{\prime} H_{2}}\left(\psi_{2}-\psi_{1}\right)+\beta y,
\end{aligned}
$$

where $q_{i}$ is the potential vorticity, $\nabla^{2}$ denotes the horizontal Laplacian operator, $d / d t \equiv \partial_{t}-\psi_{i y} \partial_{x}+\psi_{i x} \partial_{y}(i=1$ or 2) is the material derivative, and subscripts $x, y$ and $t$ denote partial differentiation. The meaning of the physical parameters is as follows: $f_{0}$ is the Coriolis parameter at the central latitude, $\beta$ is the meridional gradient of the Coriolis parameter at the same latitude, $g^{\prime}=g\left(\rho_{2}-\rho_{1}\right) / \rho_{0}$ is the reduced gravity with $g$ the acceleration due to gravity and $\rho_{0}$ the mean density. The values of these parameters were fixed at values typical of the subtropical gyre as shown in Table 2. The dissipation terms adopted here originate from horizontal diffusion of the momentum rather than of the potential vorticity. The value of the horizontal eddy viscosity $A_{\mathrm{H}}$ is chosen to be $150 \mathrm{~m}^{2} \mathrm{~s}^{-1}$. The last term on the right-hand side of (1) represents external forcing where

$$
W=-\tau_{0} \frac{\pi}{L} \sin \frac{\pi y}{L}\left(\cos \frac{2 \pi t}{T}+1\right)
$$
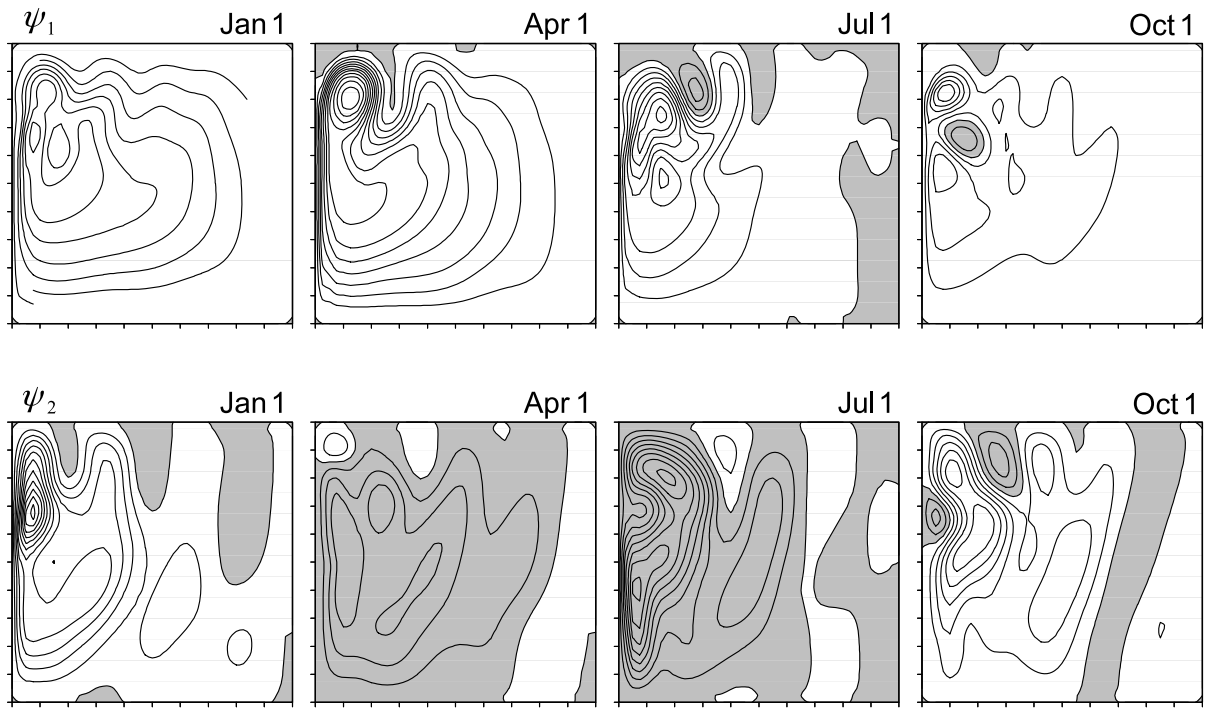

Fig. 2. Instantaneous streamfunctions $\psi_{1}$ (top) and $\psi_{2}$ (bottom) at year 801 for $\tau_{0}=0.079 \mathrm{~N} \mathrm{~m}^{-2}$, drawn at an interval of 3 months. Contour intervals are $2000 \mathrm{~m}^{2} \mathrm{~s}^{-1}$ for $\psi_{1}$ and $500 \mathrm{~m}^{2} \mathrm{~s}^{-1}$ for $\psi_{2}$. Regions where the streamfunction takes on negative values are shaded. 

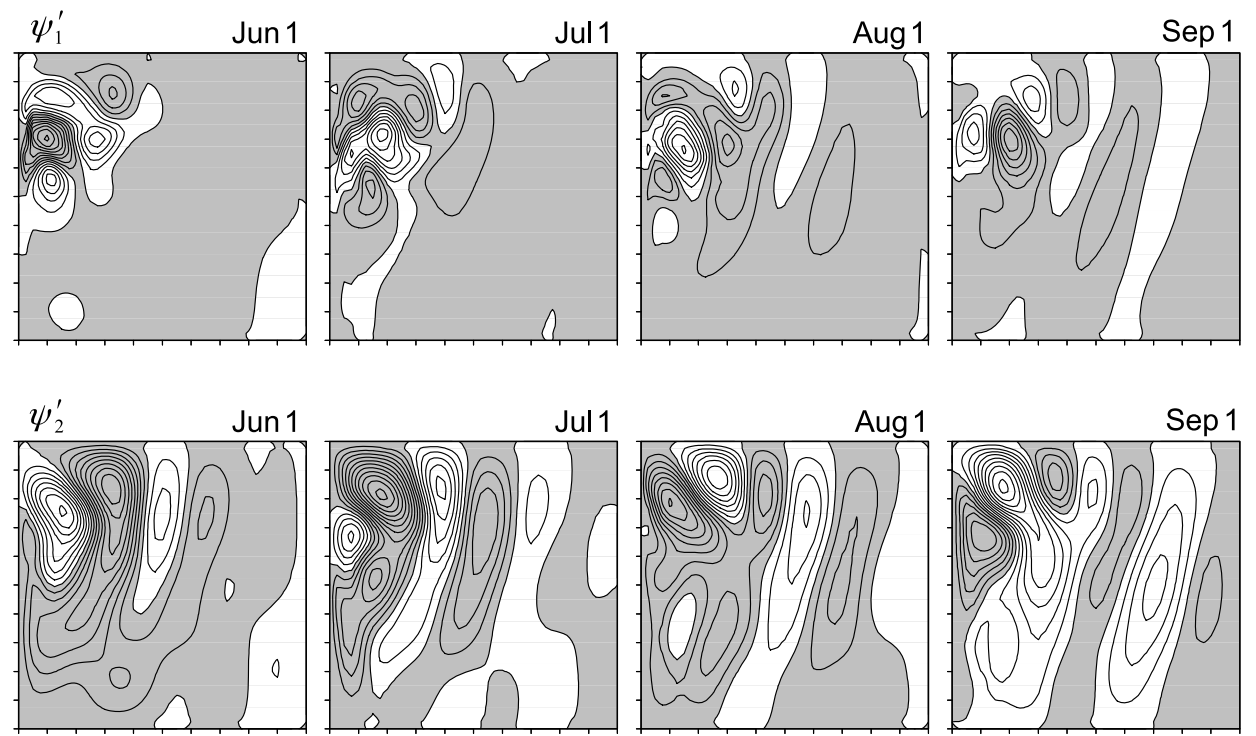

Fig. 3. Eddy components of $\psi_{1}$ (top) and $\psi_{2}$ (bottom) with respect to the 4-month running mean for $\tau_{0}=0.079 \mathrm{~N} \mathrm{~m}^{-2}$, drawn at an interval of 1 month. Contour intervals are $1000 \mathrm{~m}^{2} \mathrm{~s}^{-1}$ for $\psi_{1}$ and $250 \mathrm{~m}^{2} \mathrm{~s}^{-1}$ for $\psi_{2}$. Regions where the streamfunction takes on negative values are shaded.
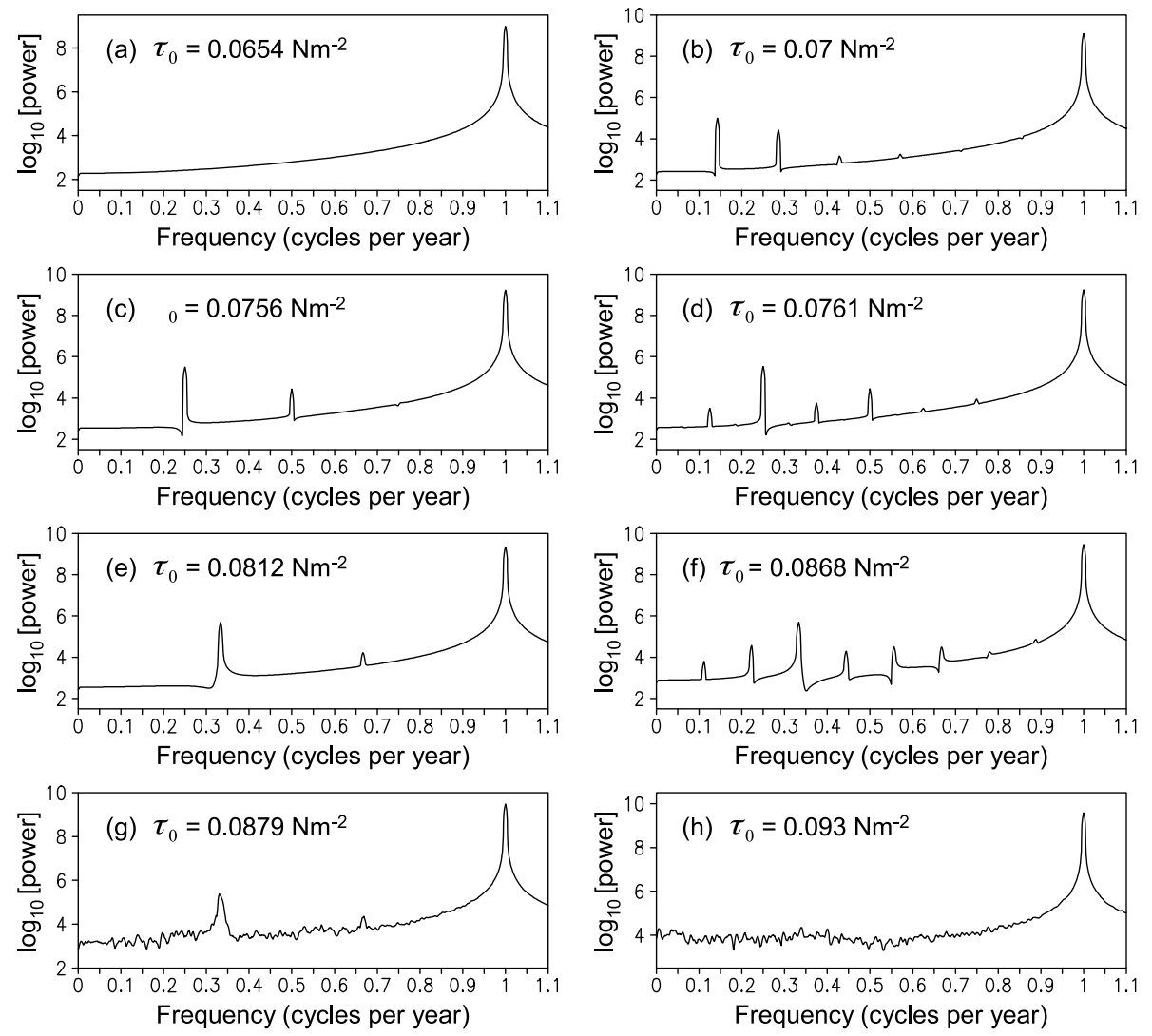

Fig. 4. Examples of power spectrum of the total energy $E$, showing (a) no peak except at the forcing frequency 1 cpy, (b) a $1 / /$ subharmonic, (c) a $1 / 4$ subharmonic, (d) a period-doubling peak at $1 / 8$ cpy which originates from the $1 / 4$ subharmonic, (e) a $1 / 3$ subharmonic, (f) a secondary $1 / 6$ subharmonic which originates from the $1 / 3$ subharmonic, $(\mathrm{g})$ a broad peak around $1 / 3 \mathrm{cpy}$, and (h) a continuous spectrum.

is the curl of the wind stress having a period of $T=1$ year, which corresponds here to 360 days; the maximum wind strength is $2 \tau_{0}$ in January and 0 in July. This forcing supplies negative vorticity directly into the upper layer over the whole basin, creating a single anticyclonic mean circulation similar to the subtropical gyre as seen later. The amplitude $\tau_{0}$ is the single control parameter in the present study.
We impose on all side boundaries the no-slip boundary condition

$$
\begin{aligned}
& \frac{\partial \psi_{1}}{\partial x}=\frac{\partial \psi_{2}}{\partial x}=0 \text { at } x=0, L, \\
& \frac{\partial \psi_{1}}{\partial y}=\frac{\partial \psi_{2}}{\partial y}=0 \text { at } y=0, L,
\end{aligned}
$$



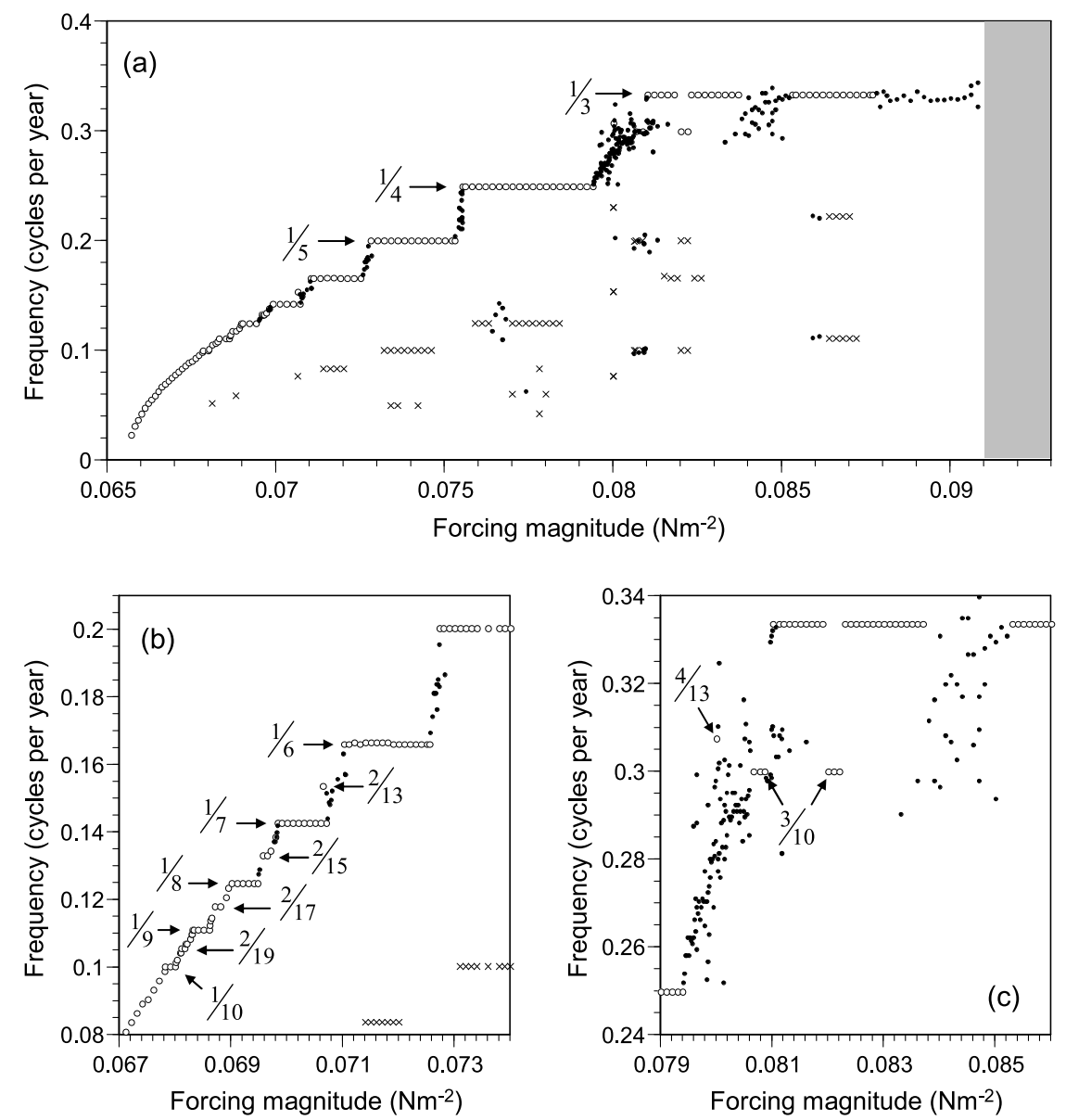

Fig. 5. Prime response frequency in the power spectrum of the total energy $E$ against the forcing magnitude $\tau_{0}$. Open circles denote sharp peaks with subharmonic or other rational frequencies, crosses denote the progeny of these subharmonics, and dots denote broad peaks. The shaded region corresponds to continuous spectra. (a) Overall view. (b, c) Enlarged view.

and the kinematic (no-normal-flow) boundary condition

$$
\psi_{1}=\psi_{2}=0 \text { at } x=0, L \text { and } y=0, L \text {. }
$$

Some authors, following McWilliams (1977) and Flierl (1977), prefer to impose the condition that each $\psi_{i}$ is a function of time at the boundaries with a global condition $\int_{0}^{L} \int_{0}^{L} \psi_{i} d x d y=0$ rather than (8) (although such manipulations lead to spurious energy fluxes across the boundaries), and it was shown that the properties of mode waves are very different when this boundary condition is used (LaCasce, 2000). We note, however, that in the present model the total volume of each layer is conserved to leading order, because the displacement of the interface has already become asymptotically zero in the QG limit. At any rate, the same kinematic condition (8) is used here as in Sakamoto (2004), and an exploration of the effects of different boundary conditions is outside the scope of the present study.

\section{Experiment}

We integrated (1)-(4) numerically on a regular grid with a resolution of $25 \mathrm{~km}$ in both $x$ and $y$ directions and a time step of $30 \mathrm{~min}$. The centered finite-difference schemes were used in both space and time. The Poisson equations for the streamfunctions were solved by the SOR method. The initial condition was assumed to be the state at rest for all values of $\tau_{0}$; the issue of hysteresis was not investigated. The
Euler-backward iteration (Haltiner and Williams, 1980) was taken every 20 time steps to damp computational modes. The impact of the latter operation on the overall evolution of our model ocean is unknown but the same procedure was applied throughout the experiment.

To verify regime shifts in the dynamical state of our model ocean when $\tau_{0}$ is changed, we recorded for each value of $\tau_{0}$ the time series of the basin-averaged potential and kinetic energy defined, respectively, by

$$
\begin{gathered}
P=\frac{\rho_{0} f_{0}^{2}}{2 g^{\prime}}\left\langle\left(\psi_{2}-\psi_{1}\right)^{2}\right\rangle, \\
K=\frac{\rho_{0}}{2} \sum_{i=1}^{2} H_{i}\left\langle\nabla \psi_{i} \cdot \nabla \psi_{i}\right\rangle,
\end{gathered}
$$

where the angle brackets denote spatial averaging over the basin. We expect that the topological and dynamic properties of our model ocean may be reproduced by using any set of relevant components of the system (e.g. Crutchfield $e t$ al., 1986). At the same time, however, care must be taken when we attempt to construct a low-dimensional attractor for a spatially extended system with many degrees of freedom (Grassberger, 1986; Procaccia, 1988). Since we cannot make a detailed spatiotemporal analysis because of the lack of available computational resources, we have chosen the global Eulerian quantities. The time series data were 

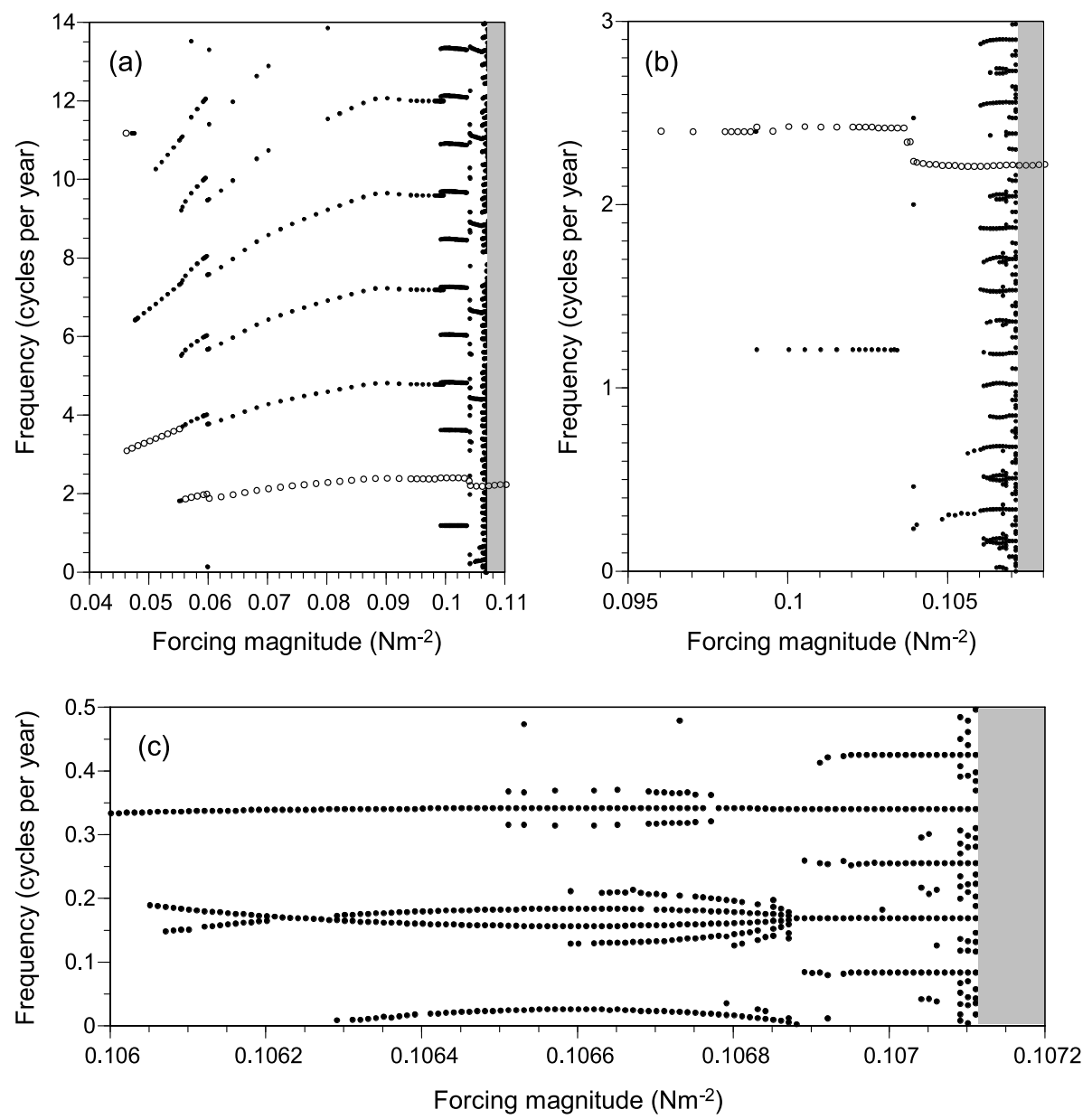

Fig. 6. The same as Fig. 5 but for the case when the wind forcing is time-independent. Open circles denote the maximum peak. The shaded region corresponds to continuous spectra. Adapted from Sakamoto (2004).

Fourier transformed to produce power spectral densities. The length of the sequence is $2^{18}$, corresponding to about 728 years, so that the frequency resolution is 0.00137 cpy (cycles per year), capable of resolving decadal variations. We have verified that transients had died away within tens of years, and therefore an 800-year integration is sufficient for the trajectories to converge to an attractor.

Before proceeding, some flow patterns are presented to check the performance of our numerical ocean. Figure 1 shows annual-mean flow fields at some year for $\tau_{0}=$ $0.079 \mathrm{~N} \mathrm{~m}^{-2}$. As mentioned in the Introduction, the timeaveraged flow in the upper layer consists of the Sverdrup interior which responds almost linearly to the wind forcing, the viscous-inertial western boundary current and the inertial recirculation located in the northwest. On the other hand, the motion in the lower layer is confined near the western boundary, counter-rotating about the central latitude, but much weaker than the upper-layer flow as in the real ocean. Figure 2 shows a seasonal change of the streamfunctions. In the upper layer the inertial recirculation survives even in summer when the wind is very weak, confirming its strong nonlinearity. The gyre in the lower layer becomes cyclonic in spring and in summer because the baroclinic adjustment is slower than the change in the wind forcing.

To make eddy activities visible, we divide the flow field $\psi$ into the mean component $\bar{\psi}$ and the eddy component $\psi^{\prime}$ as

$$
\begin{gathered}
\bar{\psi}(t)=\frac{1}{\Delta} \int_{t-\Delta / 2}^{t+\Delta / 2} \psi\left(t^{\prime}\right) d t^{\prime} \\
\psi^{\prime}(t)=\psi(t)-\bar{\psi}(t) .
\end{gathered}
$$

Hence, in discrete cases $\bar{\psi}$ is a running mean over a time interval $\Delta$ which should be chosen so as not to smooth out the mean seasonal variation. Figure 3 shows $\psi_{1}^{\prime}$ and $\psi_{2}^{\prime}$ with $\Delta=4$ months. We see that the horizontal scale of these eddies is larger than the baroclinic deformation radius $\left[g^{\prime} H_{1} H_{2} /\left(H_{1}+H_{2}\right)\right]^{1 / 2} / f_{0}(=53 \mathrm{~km}$ here $)$, and that they propagate westward, as in the case of time-independent wind forcing (e.g. Holland, 1978). In the upper layer, active eddies are dominant around the inertial recirculation, whereas in the lower layer, elongated eddies reach the southern half of the basin although their intensity is much weaker.

We have observed numerical oscillations in the southwest quadrant when either $\tau_{0}$ becomes very large or $A_{\mathrm{H}}$ is reduced (not shown). However, we have verified that no such disturbances are generated with the values of these parameters adopted in the present numerical experiments. We also confirm in Figs. 1-3 that in treating such highly nonlinear oceanic motions, grid resolution seems not to be severely 
(a) $\tau_{0}=0.07906 \mathrm{~N} \mathrm{~m}^{-2}$

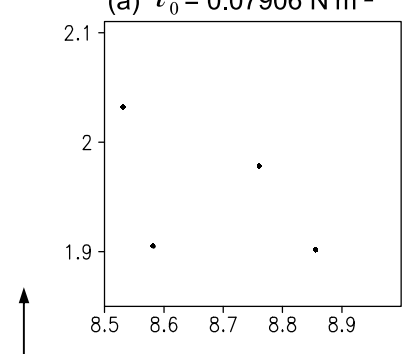

(d) $\tau_{0}=0.07962 \mathrm{~N} \mathrm{~m}^{-2}$
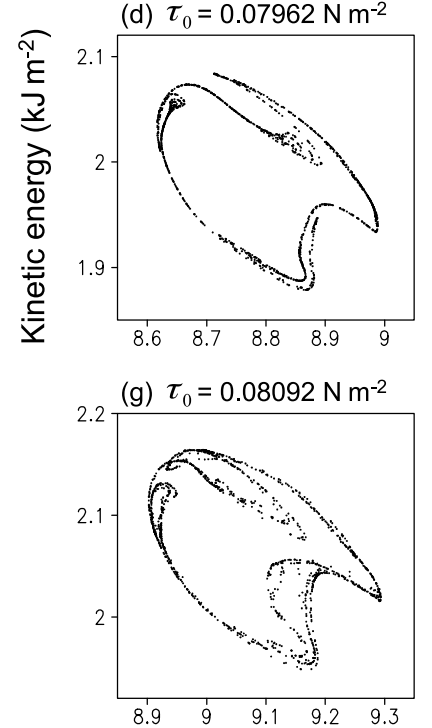

(b) $\tau_{0}=0.07939 \mathrm{~N} \mathrm{~m}^{-2}$

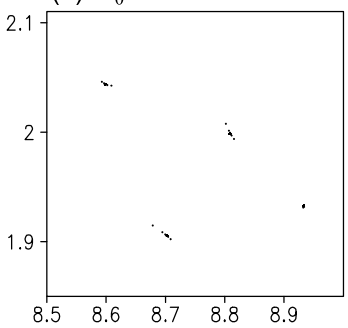

(e) $\tau_{0}=0.08002 \mathrm{~N} \mathrm{~m}^{-2}$

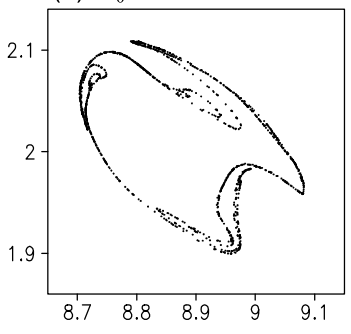

(h) $\tau_{0}=0.081 \mathrm{~N} \mathrm{~m}^{-2}$

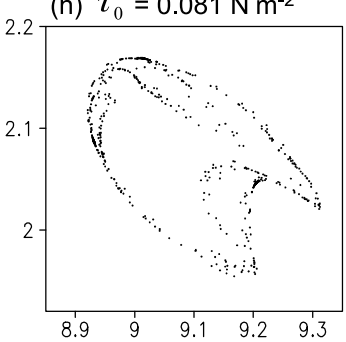

(c) $\tau_{0}=0.0794 \mathrm{~N} \mathrm{~m}^{-2}$

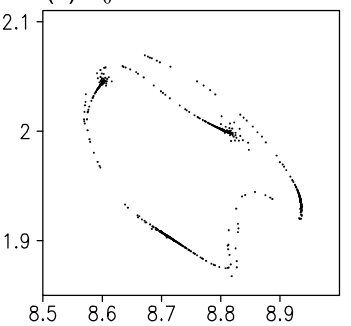

(f) $\tau_{0}=0.08054 \mathrm{~N} \mathrm{~m}^{-2}$
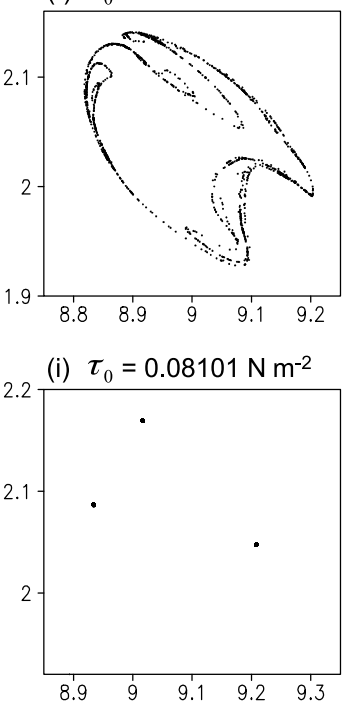

Potential energy $\left(\mathrm{kJ} \mathrm{m}^{-2}\right)$

Fig. 7. Poincaré sections $(P, K)$ at phase March 1 for various values of $\tau_{0}$, showing the transition from a 4-cycle to a 3 -cycle. The number of returns is 760. In (a) and (g) the dots are exaggerated for clarity.

restricted by the viscous scale (here, the width of the Munk layer) $\left(A_{\mathrm{H}} / \beta\right)^{1 / 3}$ and the inertial scale $(U / \beta)^{1 / 2}$ where $U$ is a velocity scale of the boundary current.

\section{Results}

Figure 4 shows the power spectrum of the total energy $E=P+K$. When the wind amplitude is sufficiently weak there are no eddies, so that we see only one peak at the forcing frequency of 1 cpy (Fig. 4(a)). The model gyre is thus totally governed by the environmental periodicity. For the stronger winds, various subharmonics appear; Fig. 4(b), (c) and (e) shows 7-, 4- and 3-cycles, respectively. Figure 4(d) and (f) exemplifies secondary bifurcation of the prime subharmonics that produces period-doubled or period-tripled components. The bottom panels correspond to aperiodic states with different spectral features. In Fig. 4(g) the peak is still distinguishable but broadens, whereas in Fig. 4(h) the spectrum in the low-frequency band ( $<1$ cpy) is noisy without any distinct peaks. It should be noted that the subharmonic peaks are much weaker than the primary peak at the forcing frequency, implying that the low-frequency signals would not be observed in calculations with a sufficiently strong stochastic component in the wind forcing.

Figure 5 summarizes the distribution of the response frequency against the forcing magnitude $\tau_{0}$ obtained from the power spectrum of $E$. For subharmonic responses, only the prime frequency is plotted with open circles and its integral multiples are omitted. Small dots denote broad peaks that are representative of chaotic behavior, and crosses stand for secondary subharmonics mentioned above. We note that the domain of the abscissa includes typical strengths of the real subtropical winds. The result should be compared with the case when the wind forcing is time-independent. The latter is shown in Fig. 6, which is a reproduction of figure 1 of Sakamoto (2004). Comparison between both cases will be made in the next section.

In Fig. 5 we observe only a basic period-1 oscillation for $\tau_{0}<0.0657 \mathrm{~N} \mathrm{~m}^{-2}$ and discern the first low-frequency components with 0.0233 cpy at $\tau_{0}=0.0657 \mathrm{~N} \mathrm{~m}^{-2}$ and 0.0316 cpy at $\tau_{0}=0.0658 \mathrm{~N} \mathrm{~m}^{-2}$. The response frequency then increases with $\tau_{0}$, but not uniformly. Various periodic $n$-cycles appear in descending order of $n$ from at least period 12 up to period 3 . We also see other rational frequencies such as $2 / 19$ cpy in much narrower subintervals. Transitions between the neighboring cycles take place through a short interval of quasiperiodic or chaotic regime, so the overall configuration resembles a devil's staircase (e.g. Devaney, 1989). However, this counterpart of the mathematical object is incomplete in the sense that there is a sudden decline around $\tau_{0}=0.0821 \mathrm{~N} \mathrm{~m}^{-2}$. We do not find a 2-cycle throughout the present calculations. Instead, the 3 -cycle directly changes to the continuous spectrum. It is unknown whether a 2-cycle cannot exist in this system or whether it is simply too unstable to appear. 
(a) $\tau_{0}=0.0835 \mathrm{~N} \mathrm{~m}^{-2}$
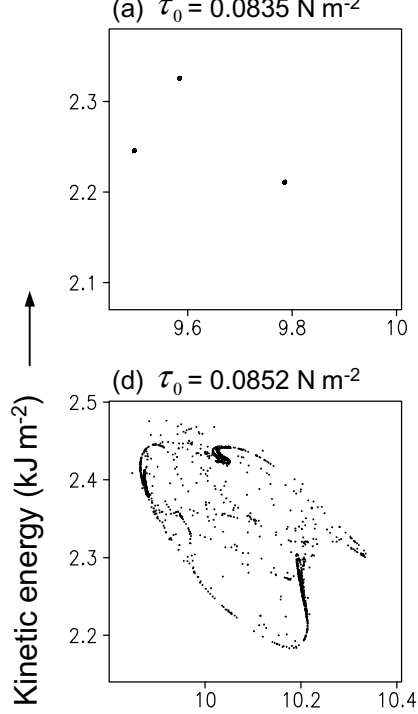

(g) $\tau_{0}=0.0874 \mathrm{~N} \mathrm{~m}^{-2}$

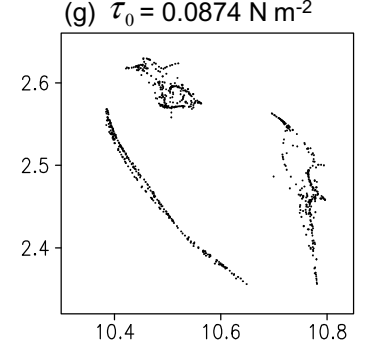

(b) $\tau_{0}=0.0836 \mathrm{~N} \mathrm{~m}^{-2}$

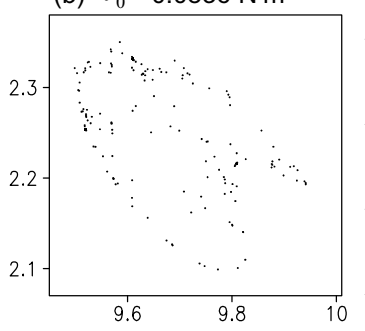

(e) $\tau_{0}=0.0854 \mathrm{~N} \mathrm{~m}^{-2}$

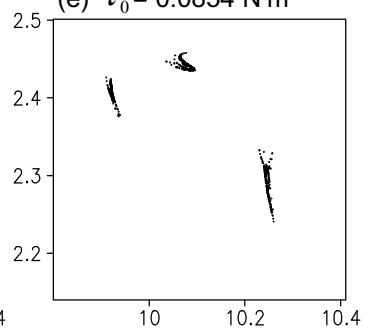

(h) $\tau_{0}=0.089 \mathrm{~N} \mathrm{~m}^{-2}$

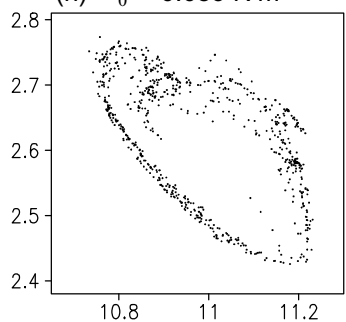

Potential energy $\left(\mathrm{kJ} \mathrm{m}^{-2}\right)$ (c) $\tau_{0}=0.0841 \mathrm{~N} \mathrm{~m}^{-2}$

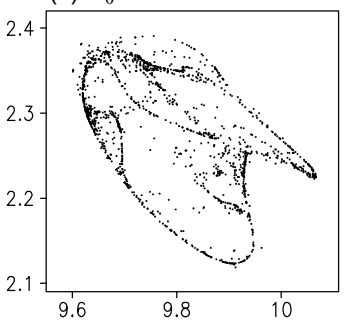

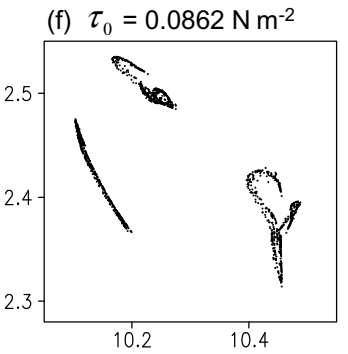

(i) $\tau_{0}=0.0928 \mathrm{~N} \mathrm{~m}^{-2}$

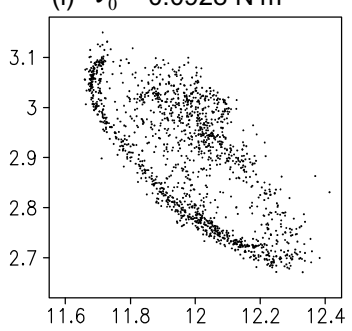

$\begin{array}{lllll}11.6 & 11.8 & 12 & 12.2 & 12.4\end{array}$

Fig. 8. The same as Fig. 7 but for larger values of $\tau_{0}$, showing the transition of a 3-cycle.

To obtain some insight into the regime shifts from a topological point of view, Fig. 7 plots $(P, K)$ at phase March 1 for various values of $\tau_{0}$. The sequence of panels shows the transition from a 4-cycle to a 3-cycle. During the transition, the equilibrium points are connected smoothly, confirming quasiperiodic or chaotic behavior (Fig. 7(c)-(h)). It seems that the two sets of fixed points are exchanged through stretching and folding, characteristic of strange attractors. A similar process was observed in the transitions between the higher-order subharmonics. However, the destabilization of a 3-cycle seems very different, as shown in Fig. 8. As $\tau_{0}$ is increased, the 3-cycle collapses abruptly (Fig. 8(b)). The points then converge not into a 2-cycle but into islands around the fixed points of the original 3-cycle (Fig. 8(c)(e)); each island looks like a chaotic attractor, although no dimensional analysis has been made here. As $\tau_{0}$ is increased further, the islands grow (Fig. 8(f), (g)), overlap to each other and eventually merge into a single scattered attractor (Fig. 8(h), (i)). Such a dispersed distribution was not seen between the other cycles.

\section{Discussion}

From the present experiment we have found that the unsteady circulation tends to be synchronized with the forcing frequency to produce the subharmonics and that the response frequency changes with the forcing amplitude in a manner similar to a devil's staircase. This is in contrast with the cases of time-independent wind forcing (Ta- ble 1), in which intrinsic low-frequency signals are observed. It is known, although there is no general consensus, that those frequencies may be attributed to the propagating plane Rossby waves or to the Rossby basin-modes. In fact, the primary frequency $11.2 \mathrm{cpy}$ at $\tau_{0}=0.046 \mathrm{~N} \mathrm{~m}^{-2}$ (Fig. 6) is very close to the eigenfrequency of the gravest barotropic basin mode, and the frequencies 2.4-cpy and 2.2cpy at $\tau_{0}>0.09 \mathrm{~N} \mathrm{~m}^{-2}$ are in good agreement with those of the higher-order barotropic or baroclinic modes (Pedlosky, 1987; Dijkstra, 2000). Such intrinsic modes do not appear in Fig. 5, indicating that they are overwhelmed by the modes which are tightly trapped by the periodic forcing. At present we are uncertain whether the entrainment is dominant over the intrinsic modes irrespective of the basin size. We should also confirm the dependence of the structure of the Arnold tongues on the eddy viscosity as well as the grid size.

The route to chaos with increasing $\tau_{0}$ is also changed dramatically with or without the periodic forcing. In the case of the time-independent wind forcing (Fig. 6) the lowfrequency variations are produced when $\tau_{0}$ becomes larger. More specifically, the primary mode becomes unstable, producing an interannual fluctuation, followed by frequency locking and successive period-doubling bifurcations that ultimately lead to the continuous spectrum. These properties are quite different from the appearance of the climbing devil's staircase in Fig. 5. We speculate that in the periodically forced ocean there is a conflict between the subhar- 
monic resonances and the Feigenbaum cascades associated with the instabilities of the large-scale waves. To estimate the relative importance of these competing tendencies we need to investigate our model ocean further by changing, among others, the forcing frequency as a second control parameter before we enter into stochastic forcing.

Finally, the main result is summarized in terms of the ocean climate. The occurrence of the $n$-cycles indicates that in our idealized ocean driven by the prevailing seasonal winds the subtropical gyre may experience interannual and decadal variations which are produced internally by the entrainment and the nonlinear processes. By contrast, it seems plausible that additional external mechanisms, such as stochastic forcing and ocean-atmosphere coupling, should be taken into account to explain interannual variations whose time scale is not commensurable with annual period. Although there is a tremendous gap between our model ocean and the real ocean, we take a step forward to suggest the role played by the nonlinearity in selecting the low-frequency variations in the North Pacific under the seasonal winds. We note that variations having time scales around 3-4 years, which in our model ocean seem stable in a wide range of wind amplitudes, are not at all special in the atmosphere and the ocean; see, for example, the path variation of the Kuroshio in the North Pacific subtropical gyre.

\section{Conclusion}

Using a two-layer quasigeostrophic model, a numerical study is made of an oceanic single-gyre circulation within a relatively small basin, forced by annually periodic winds. As the forcing amplitude is increased, various subharmonics appear in the time series of the total energy (and other local variables). The response frequency of these periodic regimes, together with much narrower quasiperiodic and chaotic regimes, constitutes a quasi-devil's staircase. Thus, our idealistic ocean, viewed as a nonlinear dynamical system, acts as a frequency divider. An immediate implication is that large-scale motions having interannual and decadal time scales may be produced by internal nonlinear processes even under the predominant seasonal winds.

Acknowledgments. I wish to thank Profs. Gurbax S. Lakhina and Yoshiharu Omura and for the opportunity to present my work in the special session Nonlinear Processes in Earth and Planetary Science in the 2005 Joint Meeting for Earth and Planetary Science.

\section{References}

Anderson, D. L. T. and P. D. Killworth, Non-linear propagation of long Rossby waves, Deep-Sea Res., 26, 1033-1050, 1979.

Auad, G., Interdecadal dynamics of the North Pacific Ocean, J. Phys. Oceanogr., 33, 2483-2503, 2003.

Berloff, P. S. and S. Meacham, The dynamics of an equivalent-barotropic model of the wind-driven circulation, J. Mar. Res., 55, 407-451, 1997.

Berloff, P. S. and S. Meacham, The dynamics of a simple baroclinic model of the wind-driven circulation, J. Phys. Oceanogr., 28, 361-388, 1998.

Berloff, P. S. and J. C. McWilliams, Large-scale, low-frequency variability in wind-driven ocean gyres, J. Phys. Oceanogr., 29, 1925-1949, 1999.

Cessi, P. and S. Louazel, Decadal oceanic response to stochastic wind forcing, J. Phys. Oceanogr., 31, 3020-3029, 2001.

Chang, K.-I., M. Ghil, K. Ide, and C.-C. A. Lai, Transition to aperiodic variability in a wind-driven double-gyre circulation model, J. Phys. Oceanogr., 31, 1260-1286, 2001.
Crutchfield, J., J. D. Farmer, N. Packard, and R. Shaw, Chaos, Sci. Amer., 255, 46-57, 1986.

Deser, C., Evidence for a wind-driven intensification of the Kuroshio Current Extension from the 1970s to the 1980s, J. Clim., 12, 1697-1706, 1999.

Devaney, R. L., An Introduction to Chaotic Dynamical Systems 2nd ed., 336 pp., Addison-Wesley, Redwood City, CA, 1989.

Dewar, W. K., Arrested fronts, J. Mar. Res., 49, 21-55, 1991.

Dijkstra, H. A., Nonlinear Physical Oceanography, 456 pp., Kluwer, Dordrecht, 2000.

Dijkstra, H. A. and C. A. Katsman, Temporal variability of the wind-driven quasi-geostrophic double gyre ocean circulation: Basic bifurcation diagrams, Geophys. Astrophys. Fluid Dyn., 85, 195-232, 1997.

Dijkstra, H. A. and W. Weijer, Stability of the global ocean circulation: Basic bifurcation diagrams, J. Phys. Oceanogr., 35, 933-948, 2005.

Flierl, G. R., Simple applications of McWilliams "A note on a consistent quasi-geostrophic model in a multiply connected domain," Dyn. Atmos. Oceans, 1, 443-453, 1977.

Frankignoul, C., P. Müller, and E. Zorita, A simple model of the decadal response of the ocean to stochastic wind forcing, J. Phys. Oceanogr., 27, 1533-1546, 1997.

Gent, P. R. and J. C. McWilliams, Isopycnal mixing in ocean circulation models, J. Phys. Oceanogr., 20, 150-155, 1990.

Ghil, M., Y. Feliks, and L. U. Sushama, Barotropic and baroclinic aspects of the wind-driven ocean circulation, Physica D, 167, 1-35, 2002.

Grassberger, P., Do climatic attractor exist?, Nature, 323, 609-612, 1986.

Hasselmann, K., Stochastic climate models. I. Theory, Tellus, 28, 473-485, 1976.

Haltiner, G. J. and R. T. Williams, Numerical Prediction and Dynamic Meteorology 2nd ed., 477 pp., John Wiley \& Sons, New York, 1980.

Hendershott, M. C., Single layer models of the general circulation, in General Circulation of the Ocean, edited by D. I. Abarbanel and W. R. Young, 291 pp., Springer-Verlag, New York, 1987.

Holland, W. R., The role of mesoscale eddies in the general circulation of the ocean-numerical experiments using a wind-driven quasigeostrophic model, J. Phys. Oceanogr., 8, 363-392, 1978.

Holland, W. R. and L. B. Lin, On the generation of mesoscale eddies and their contribution to the oceanic general circulation. I. A preliminary numerical experiment, J. Phys. Oceanogr., 5, 642-657, 1975.

Holland, W. R., D. E. Harrison, and A. J. Semtner, Jr., Eddy-resolving numerical models of large-scale ocean circulation, in Eddies in Marine Science, edited by A. R. Robinson, 609 pp., Springer-Verlag, New York, 1983.

Jiang, S., F.-F. Jin, and M. Ghil, Multiple equilibria, periodic, and aperiodic solutions in a wind-driven, double gyre, shallow-water model, J. Phys. Oceanogr., 25, 764-786, 1995.

Kamenkovich, V. M., V. A. Sheremet, A. R. Pastushkov, and S. O. Belotserkovsky, Analysis of the barotropic model of the subtropical gyre in the ocean for finite Reynolds numbers. Part I, J. Mar. Res., 53, 959-994, 1995.

LaCasce, J. H., Baroclinic Rossby waves in a square basin, J. Phys. Oceaongr., 30, 3161-3178, 2000.

Lorenz, E. N., Available potential energy and the maintenance of the general circulation, Tellus, 7, 157-167, 1955.

Luyten, J. R., J. Pedlosky, and H. Stommel, The ventilated thermocline, J. Phys. Oceanogr., 13, 292-309, 1983.

McCalpin, J. D. and D. B. Haidvogel, Phenomenology of the lowfrequency variability in a reduced-gravity, quasigeostrophic double-gyre model, J. Phys. Oceanogr., 26, 739-752, 1996.

McWilliams, J. C., A note on a consistent quasigeostrophic model in a multiply connected domain, Dyn. Atmos. Oceans, 1, 427-441, 1977.

Meacham, S. P., Low-frequency variability in the wind-driven circulation, J. Phys. Oceanogr., 30, 269-293, 2000.

Meacham, S. P. and P. S. Berloff, Barotropic, wind-driven circulation in a small basin, J. Mar. Res., 55, 523-563, 1997.

Miller, A. J., D. R. Cayan, T. P. Barnett, N. E. Graham, and J. M. Oberhuber, Interdecadal variability of the Pacific Ocean: model response to observed heat flux and wind stress anomalies, Clim. Dyn., 9, 287-302, 1994.

Nauw, J. J. and H. A. Dijkstra, The origin of low-frequency variability of double-gyre wind-driven flows, J. Mar. Res., 59, 567-597, 2001.

Orlanski, I., The influence of bottom topography on the stability of jets in a baroclinic fluid. J. Atmos. Sci., 26, 1216-1232, 1969.

Pedlosky, J., Geophysical Fluid Dynamics 2nd ed., 710 pp., SpringerVerlag, New York, 1987.

Pedlosky, J., The dynamics of the oceanic subtropical gyres, Science, 248, 
316-322, 1990.

Pierce, D. W., T. P. Barnett, N. Schneider, R. Saravanan, D. Dommenget, and M. Latif, The role of ocean dynamics in producing decadal climate variability in the North Pacific, Clim. Dyn., 18, 51-70, 2001.

Primeau, F., Multiple equilibria and low-frequency variability of the winddriven ocean circulation, J. Phys. Oceanogr., 32, 2236-2256, 2002.

Procaccia, I., Complex or just complicated?, Nature, 333, 498-499, 1988.

Qiu, B., Kuroshio Extension variability and forcing of the Pacific decadal oscillations: Responses and potential feedback, J. Phys. Oceanogr., 33, 2465-2482, 2003.

Qiu, B. and T. M. Joyce, Interannual variability in the mid- and lowlatitude western North Pacific, J. Phys. Oceanogr., 22, 1062-1079, 1992.

Rhines, P. B. and W. R. Young, A theory of the wind-driven circulation I. Mid-ocean gyres, J. Mar. Res., 40 (Suppl.), 559-596, 1982.

Sakamoto, T., On discontinuities in the Sverdrup interior, J. Phys. Oceanogr., 29, 2457-2461, 1999.

Sakamoto, T., Determination of wind-driven ocean circulation inside closed characteristics, Geophys. Astrophys. Fluid Dyn., 94, 151-176, 2001.

Sakamoto, T., Western boundary current separation caused by a deep countercurrent, Geophys. Astrophys. Fluid Dyn., 96, 179-199, 2002.

Sakamoto, T., A route to Eulerian chaos in a two-layer wind-driven ocean, Fluid Dyn. Res., 34, 117-134, 2004.
Schneider, N., A. J. Miller, and D. W. Pierce, Anatomy of North Pacific decadal variability, J. Clim., 15, 586-605, 2002.

Simonnet, E. and H. A. Dijkstra, Spontaneous generation of low-frequency modes of variability in the wind-driven ocean circulation, J. Phys. Oceanogr., 32, 1747-1762, 2002.

Sura, P., F. Lunkeit, and K. Fraedrich, Decadal variability in a simplified wind-driven ocean model, J. Phys. Oceanogr., 30, 1917-1930, 2000.

Thompson, L. and C. A. Ladd, The response of the North Pacific Ocean to decadal variability in atmospheric forcing: Wind versus buoyancy forcing, J. Phys. Oceanogr., 34, 1373-1386, 2004.

Trenberth, K. E. and J. W. Hurrell, Decadal atmosphere-ocean variations in the Pacific, Clim. Dyn., 9, 303-319, 1994.

van der Vaart, P. C. F., H. M. Schuttelaars, D. Calvete, and H. A. Dijkstra, Instability of time-dependent wind-driven ocean gyres, Phys. Fluids, 14 3601-3615, 2002.

Willmott, A. J., A note on the steepening of long Rossby waves, Deep-Sea Res., 32, 613-617, 1985.

Young, W. R., Baroclinic theories of the wind driven circulation, in General Circulation of the Ocean, edited by D. I. Abarbanel and W. R. Young, 291 pp., Springer-Verlag, New York, 1987.

T. Sakamoto (e-mail: sakamoto@eps.s.u-tokyo.ac.jp) 